\title{
Analysis of a frequency divider by two based on a differential nonlinear transmission line
}

\author{
Mabel Pontón, Almudena Suárez \\ University of Cantabria, Santander, Spain
}

\begin{abstract}
A recently proposed frequency divider by two, based on differential nonlinear transmission line, acting like a reflective distributed resonator, is analyzed in-depth. The flip bifurcation locus of a single cell is obtained analytically, which enables an understanding of the divider behavior and an initial estimation of its element values. The possibility to modify the division threshold and bandwidth through the proper selection of an additional linear capacitor is demonstrated. The influence of the number of cells on the division bandwidth and on the generated standing wave at the subharmonic frequency is also investigated. The techniques have been applied to two frequency divider with 1.5 and $2.2 \mathrm{GHz}$ input frequency.

Index Terms - Nonlinear transmission line, frequency divider, stability, bifurcation.
\end{abstract}

\section{INTRODUCTION}

The works [1-2] propose a frequency divider topology based on the use of a differential nonlinear transmission line (NLTL). The differential line behaves like a reflective distributed resonator, such that the subharmonic frequency terminates in virtual ground at both ends and the output signal(s) is extracted from internal cells by means of a buffer. The subharmonic component is sustained by the gain exhibited by the varactors under the pump signal at $2 \omega$ and, in this manner, a symmetric standing wave is formed through the distributed resonator. The reflective structure with distributed gain stages enables the frequency division from low input power and, compared with transistor-based dividers, has two main advantages [2]: no static power consumption and low phase-noise spectral density. Unlike injection-locked dividers [3], where the noise spectrum approaches that of the freerunning oscillation from certain frequency offset, the phase noise follows that of the input signal up to the noise floor.

As shown in [1-2], the gain increase with the number of NLTL cells requires a minimization of the dispersion effect, that is, the pump propagation constant at $2 \omega$, denoted $\beta_{2}$, must be approximately twice the propagation constant at $\omega$. To facilitate this relationship, an additional capacitor $c_{c}$, connected as shown in Fig. 1, is proposed in [2], which decreases the average capacitance at $2 \omega$. The work [2] departs from a continuous transmission line analysis which is modified in a mostly qualitative manner when considering the realistic discrete case. Here a single division cell is analytically formulated to investigate the dependence of the division threshold and bandwidth on the capacitor $c_{c}$. The analytical formulation provides insight into the particular shape of the flip bifurcation loci [4-5] obtained through the variation of critical design parameters. It also enables an initial estimation of the circuit element values required in the multicell divider, which is analyzed numerically with specific harmonic balance $(\mathrm{HB})$ tools. For validation, two dividers with $N=4$ and $N=6$ cells have been manufactured and experimentally characterized.

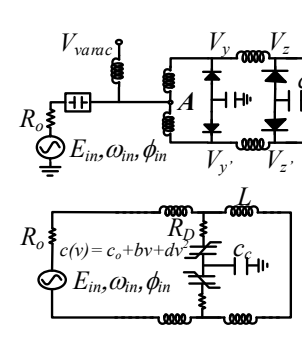

(b)

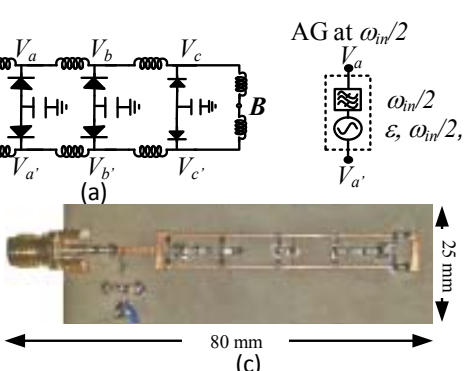

(c)
Fig. 1 Diode-based frequency divider, with an AG in a differential connection. (a) Multi-cell divider. (b) Single cell. (c) Photograph.

\section{EFFECT OF THE DISPERSION-COMPENSATION CAPACITOR}

Initially, the single cell divider of Fig. 1(b) will be considered. The diode voltage is approximated as:

$$
v(t)=V e^{j \omega t}+V e^{-j \omega t}+V_{2} e^{j\left(2 \omega t+\phi_{2}\right)}+V_{2} e^{-j\left(2 \omega t+\phi_{2}\right)}
$$

where $\omega=\omega_{i n} / 2$ is the subharmonic frequency and the phase origin is taken at $\omega$. Considering a second-order Taylor series expansion of the varactor capacitance, $c(v)=c_{o}+b v+d v^{2}$, the current phasors at $\omega$ and $2 \omega$ are:

$$
\begin{aligned}
& I_{1}=\left[j \omega\left(c_{o}+d V^{2}+2 d V_{2}^{2}+b V_{2} \cos \phi_{2}\right)-\omega b V_{2} \sin \phi_{2}\right] V \\
& I_{2}=j \omega\left(\left(2 C_{o} V_{2}+4 d V^{2} V_{2}\right) e^{j \phi_{2}}+b V^{2}\right)
\end{aligned}
$$

Due to the circuit symmetry and assuming an odd mode subharmonic oscillation $\left(180^{\circ}\right.$ phase shift between the two branches) the middle points $A$ and $B$ are virtual short circuits at the subharmonic frequency. The point $B$ is a virtual open circuit at the input frequency $2 \omega$. Considering also the input equivalent network at $2 \omega$, one obtains the following system:

$$
\begin{aligned}
& V+\left(R_{D}+j \frac{L \omega}{2}\right) I_{1}=0 \\
& V_{2} e^{j \phi_{2}}+Z_{T}(\omega) I_{2}-E_{i n} e^{j \phi_{i n}}=0
\end{aligned}
$$


where $\quad Z_{T}=R_{T}+j\left[L \omega_{i n}-2 /\left(c_{c} \omega_{i n}\right)\right] \quad, \quad R_{T}=2 R_{o}+R_{D}$ $R_{o}=50 \Omega, R_{D}$ is the diode resistance, $E_{i n}$ is the input voltage and $\phi_{i n}$ is the opposite of the node voltage phase shift. At the flip bifurcation [4-5] where the subharmonic component arises, the above steady-state conditions are fulfilled for $V \rightarrow 0$ [5]. Replacing this condition into (3) (a) and splitting into real and imaginary parts, one obtains:

$$
\begin{aligned}
& -\frac{\omega_{\text {in }}}{2} b V_{2} \sin \phi_{2}+\frac{R_{D}}{R_{D}^{2}+\left(L \omega_{\text {in }} / 4\right)^{2}}=0 \\
& \frac{\omega_{\text {in }}}{2}\left(c_{o}+b V_{2} \cos \phi_{2}\right)-\frac{L \omega_{i n} / 4}{R_{D}^{2}+\left(L \omega_{i n} / 4\right)^{2}}=0
\end{aligned}
$$

where a moderate input amplitude has been assumed, thus neglecting $V_{2}^{2}$ in (2). From (4) one can calculate voltage component $V_{2 o} e^{j \phi_{2 o}}$ at the flip bifurcation:

$$
\begin{aligned}
V_{2 o} \sin \phi_{2 o} & =\frac{2 R_{D}}{\omega_{i n} b\left[R_{D}^{2}+\left(L \omega_{i n} / 4\right)^{2}\right]} \\
V_{2 o} \cos \phi_{2 o} & =\frac{L \omega_{i n} / 2}{\omega_{i n} b\left[R_{D}^{2}+\left(L \omega_{i n} / 4\right)^{2}\right]}-\frac{c_{o}}{b}
\end{aligned}
$$

To the approximation order considered here, the voltage $V_{2 o} e^{j \phi_{2 o}}$ at the flip bifurcation does not depend on $E_{i n}$. The division threshold $E_{\text {ino }}$ can be directly obtained by replacing $V_{2 o}$ into (3)(a):

$$
E_{\text {ino }}=V_{2 o}\left\{\left[1-\left(L c_{o} \omega_{i n}^{2}-c_{0} / c_{p}\right)\right]^{2}+\left(R_{T} c_{o} \omega_{i n}\right)^{2}\right\}^{1 / 2}
$$

where $c_{p}=c_{c} / 2$. Expression (6) provides insight into the effect of $c_{c}$ [which only affects (6)] on the division threshold $E_{\text {ino. }}$. It enables the reduction of $E_{\text {ino }}$ for given values of $L$ and the diode parameters. For illustration, the varactor diode SMV1231 and inductor $L=5.8 \mathrm{nH}$ will be considered, at the input frequency $f_{\text {in }}=4.34 \mathrm{GHz}$. Fig. 2 presents the variation of $E_{\text {ino }}$ with $c_{c}$ calculated with (6). The curve constitutes a flip bifurcation locus, or division boundary, and the division region (above the locus) is indicated in the figure. The minimum division threshold is obtained for $c_{p m}=-c_{o} /\left(1-L c_{o} \omega_{i n}^{2}\right)$, as deduced from (6). For higher number of cells, subharmonic gain increases, which should lead to higher amplitude at $\omega_{\text {in }} / 2$. Unlike [2], the configuration in this work (Fig. 1) is terminated in inductors, instead of varactors, as otherwise an additional transmission line section is required to avoid short-circuiting the outermost NLTL diodes. The multi-cell divider can be analyzed only through numerical simulation. Here harmonic balance (HB) is used, together with an auxiliary generator (AG) [4] at
$\omega_{A G}=\omega_{i n} / 2$, with phase $\phi_{A G}=0$ (phase origin), connected in differential manner [Fig. 1(a)]. Full diode models and $N H=20$ harmonic terms are considered, with the design departing from the $L$ and $c_{c}$ values obtained with the analytical formulation. For the flip bifurcation detection, the AG amplitude is set to small value, $A_{A G}=\varepsilon$ solving the non-perturbation condition $Y_{A G}\left(c_{c}, \phi_{i n}, E_{i n}\right)=0$ (with the pure HB system as inner tier), which provides a curve in the plane $\left(c_{c}, E_{i n}\right)$. The flip bifurcation loci resulting for $N=4$ and $N=6$ have been represented in Fig. 2. The shape is similar to the one provided by (6), with a well-defined minimum. HB simulation with $N=1$ and $N H=2$ is overlapped with (6). The division threshold decreases with $N$, due to the action of more subharmonic gain stages. The variation of the subharmonic amplitude trough the NLTL cells has been analyzed in Fig. 3 for three different $N$ values. A symmetric stationary wave is formed at the subharmonic frequency [Fig. 3(a)], terminated in voltage minima due to the virtual short-circuits resulting from the differential operation.

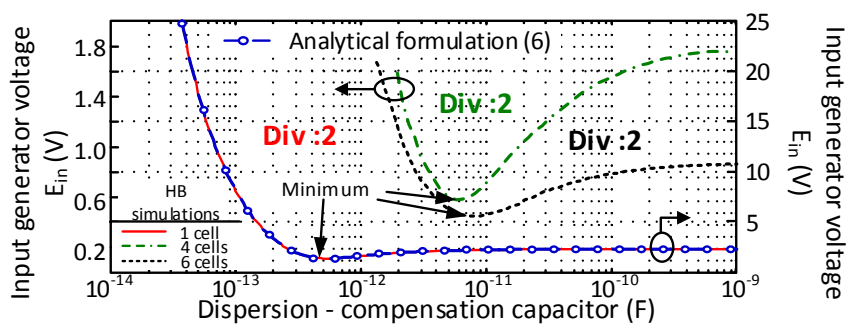

Fig. 2 Variation of the bifurcation threshold $E_{\text {ino }}$ (in single-sided spectrum) with $c_{c}$. Results of (6) are compared with HB simulations for $N=1, N=4$ and $N=6$.

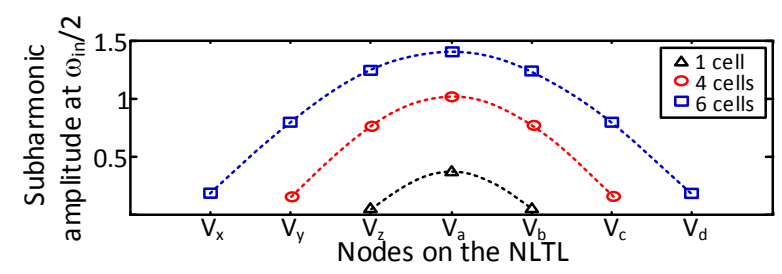

Fig. 3 Amplitude distributions for two different numbers of cells $(N=4$ and $N=6)$ with $E_{i n}=0.7 \mathrm{~V}$.

\section{ANALYSIS OF THE OPERATION BANDWIDTH}

For a single cell and constant $E_{i n}$, the flip bifurcation locus in terms of $\omega_{i n}$ and $c_{c}$, is obtained by solving (6) for $c_{p}$ :

$$
c_{p}=c_{c} / 2=\frac{c_{o}}{L c_{o} \omega_{i n}^{2}-1 \pm \sqrt{\left(E_{\text {in }} / V_{2 o}\right)^{2}-R_{T}^{2} c_{o}^{2} \omega_{i n}^{2}}}
$$

Sweeping $\omega_{i n}$ two different sections are obtained, respectively corresponding to the positive and negative sign before the root operation. They have been traced in solid and 
dashed lines in Fig. 4, for three different $E_{\text {in }}$ values. The division region is inside each flip-bifurcation locus. For too low $c_{c}$, the diode approaches an open-circuit situation at $\omega_{i n}$, which justifies the $c_{c}$ minimum. Too high $c_{c}$ has no impact on the circuit behavior. The edges of the maximum frequency bandwidth are given by the condition $\left[E_{\text {in }} / V_{2 o}\left(\omega_{i n}\right)\right]^{2}-R_{T}^{2} c_{o}^{2} \omega_{i n}^{2}=0$ and correspond to the same $c_{c}$, calculated from (7): $\quad c_{c M}=2 c_{o} /\left(L c_{o} \omega_{i n}^{2}-1\right) \quad$. This capacitance, providing the maximum bandwidth, does not depend on $E_{i n}$, as validated with the analysis in Fig. 4.

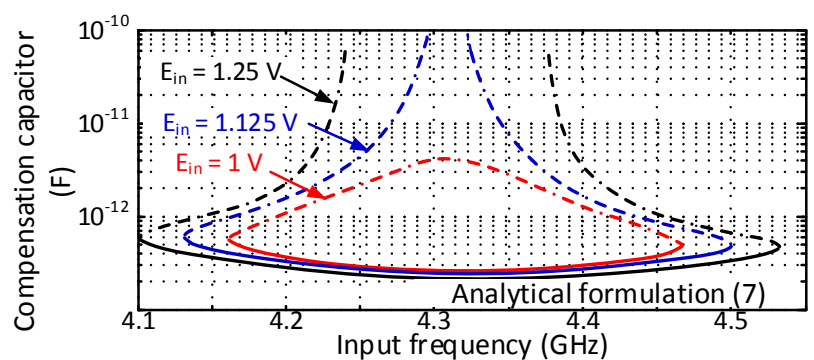

Fig. 4 Flip bifurcation loci obtained with (7) for three $E_{\text {in }}$ values.

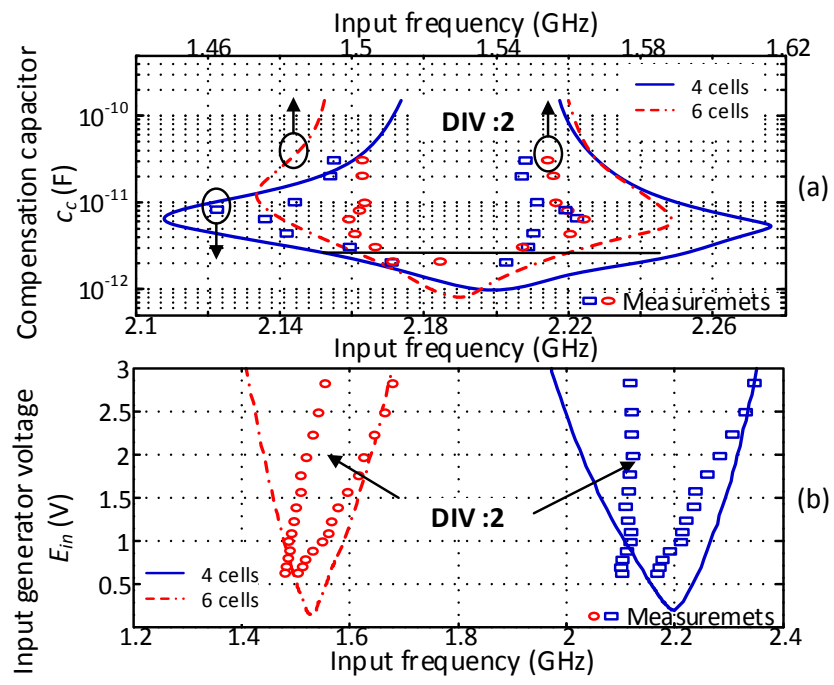

Fig. 5 Division bandwidth. (a) Plane $\omega_{i n}, c_{c}$ at $E_{i n}=1 \mathrm{~V}$. (b) Plane $\omega_{\text {in }}, E_{\text {in }}$ at $c_{c}=6.5 \mathrm{pF}$. Measurements are superimposed.

For $N=4$ and $N=6$, the circuit has been simulated in HB. The flip loci in Fig. 5(a) has been obtained solving $Y_{A G}\left(\phi_{i n}, C_{c}, E_{i n}\right)=0$, at for $E_{i n}=1 \mathrm{~V}$. The loci have the same qualitative shape as the one obtained with (7), with the lower and upper maximum frequency deviations obtained for approximately the same $c_{c}$. Measurements are superimposed with discrepancies attributed to the parasitics of the packaged $c_{c}$. The bandwidth decreases with $N$, as evidenced by the bifurcation loci $Y_{A G}\left(\phi_{i n}, \omega_{i n}, E_{i n}\right)=0$ traced in Fig. 5(b) for $c_{c}=6.5 \mathrm{pF}$. The bandwidth reduction is in agreement with the decrease in the cut-off frequency of the low-pass configuration. Unlike injection-locked dividers, there is a $P_{\text {in }}$ threshold for divider operation and the circuit does not oscillate outside the division bandwidth, which can be advantageous. Finally, the divided solution has been calculated solving for $Y_{A G}\left(\phi_{i n}, A_{A G}\right)=0$ at $E_{\text {in }}=1 \mathrm{~V}$. Fig. 6 shows the simulated and measured waveforms through the NLTL cells. Non-symmetric waveforms are due to the unequal terminations at the even harmonics terms. As expected, maximum amplitude is obtained at the middle cell.


(a)

(b)

Fig. 6 Differential voltage waveforms through the 6 -cell NLTL frequency divider. (a) Simulations. (b) Measurements with the oscilloscope DSO90804A. The input signal is also represented.

\section{CONCLUSION}

A recently proposed frequency divider based on a differential NLTL has been investigated. An analytical formulation of a single cell has provided insight into the circuit division threshold and frequency bandwidth. The influence of the number of cells has been analysed with harmonic balance, using an auxiliary generator in differential connection. Good agreement has been found with measurement results.

\section{ACKNOWLEDGEMENT}

Spanish project TEC2011-29264-C03-01 for financial support

\section{REFERENCES}

[1] W. Lee, E. Afshari, "Low-Noise Parametric Resonant Amplifier," IEEE Trans. Circuits and Systems I, vol.58, no.3, pp.479-492, Mar., 2011.

[2] W. Lee and E. Afshari, "Distributed Parametric Resonator: A Passive CMOS Frequency Divider," IEEE J. Solid-State Circuits, vol.45, no.9, pp.1834-1844, Sept., 2010.

[3] F. Ramírez, M. Pontón, S. Sancho, A. Suárez, "Phase-Noise Analysis of Injection-Locked Oscillators and Analog Frequency Dividers", IEEE Trans. Microw. Theory Techn., vol. 56, no.2, pp. 393-407, 2008.

[4] J. Guckehnehimer and P. Holmes, Nonlinear Oscillations, Dynamical Systems, and Bifurcations of Vector Fields, Springer Verlag, NY, 1983.

[5] A. Suárez, Analysis and Design of Autonomous Microwave Circuits. Hoboken, NJ: Wiley IEEE Pres, 2009. 